\title{
Critical Role of the Solvent Environment in Galectin-1 Binding to the Disaccharide Lactose $^{\dagger}$
}

\author{
Santiago Di Lella, ${ }^{\ddagger, \S}$, Lu Ma, ${ }^{\perp}$ Juan C. Díaz Ricci, ${ }^{\ddagger}$ Gabriel A. Rabinovich, ${ }^{\#}$ Sanford A. Asher, ${ }^{\perp}$ and \\ R. María S. Álvarez*,\$,
}

Instituto Superior de Investigaciones Biológicas (CONICET-UNT) and Instituto de Química-Física, Facultad de Bioquímica, Química y Farmacia, Universidad Nacional de Tucumán, S. M. de Tucumán, Tucumán, Argentina, Departamento de Química Inorgánica, Analítica y Química-Física, Facultad de Ciencias Exactas y Naturales, Universidad de Buenos Aires, Buenos Aires, Argentina, Department of Chemistry, Chevron Science Center, University of Pittsburgh, Pittsburgh, Pennsylvania 15260, and Laboratorio de Inmunopatología, Instituto de Biología y Medicina Experimental, CONICET, Buenos Aires, Argentina

Received September 30, 2008; Revised Manuscript Received December 4, 2008

\begin{abstract}
Galectin-1 (Gal-1), a member of a family of evolutionarily conserved glycan-binding proteins, binds specifically to poly- $N$-acetyllactosamine-enriched glycoconjugates. Through interactions with these glycoconjugates, this protein modulates inflammatory responses and contributes to tumor progression and immune cell homeostasis. The carbohydrate recognition domain includes the single protein tryptophan (Trp68). UV resonance Raman spectroscopy and molecular dynamic simulation were used to examine the change in the environment of the Trp on ligand binding. The UV Raman spectra and the calculated water radial distribution functions show that, while no large structural changes in the protein follow lactose binding, substantial solvent reorganization occurs. These new insights into the microscopic role of water molecules in Gal-1 binding to its specific carbohydrate ligands provides a better understanding of the physicochemical properties of Gal-1-saccharide interactions, which will be useful for the design of synthetic inhibitors for therapeutic purposes.
\end{abstract}

It is widely known that interactions and binding between biomolecules involve solvent redistribution. Water molecules which are tightly associated with the binding surface occupy specific positions and orientations and must vacate their positions to allow a proper binding. During the past few years, we focused our interest in studying the structural and dynamic properties of galectin-1 (Gal-1) $)^{1}$ involving binding to specific carbohydrates ligands.

Gal-1, a $\beta$-galactoside-binding protein, widely expressed in the animal kingdom, is a polypeptide containing 134 amino acids, which exist in a reversible monomer-dimer equilibrium $(1,2)$. This glycan-binding protein has been shown to play an important role in cell growth regulation and differentiation (3), and most recently, it has been shown to be involved in the modulation of innate and adaptive immune

$\dagger$ This work was partially supported by PIP $\mathrm{N}^{\circ} 6059$ of CONICET and PICT2004 N ${ }^{\circ} 21601$ of the Agencia Nacional de Promoción Científica y Tecnológica in Argentina and by NIH Grant GM8RO1EB002053. S.D.L. is a doctoral fellow of CONICET and a Fulbright and Fundación Bunge \& Born Junior Researcher. J.C.D.R., R.M.S.A., and G.A.R. are members of the research career of CONICET in Argentina.

* To whom correspondence should be addressed. Telephone: +54 381 4213226. Fax: +54 381 4248921. E-mail: mysuko@fbqf.unt.edu.ar.

${ }^{\ddagger}$ Instituto Superior de Investigaciones Biológicas (CONICET-UNT), Universidad Nacional de Tucumán.

§ Instituto de Química-Física, Facultad de Bioquímica, Química y Farmacia, Universidad Nacional de Tucumán.

"Universidad de Buenos Aires.

${ }^{\perp}$ University of Pittsburgh.

${ }^{*}$ CONICET

${ }^{1}$ Abbreviations: Gal-1, galectin-1; CRD, carbohydrate recognition domain; UVRR, ultraviolet resonance Raman; MD, molecular dynamics; ws, water site(s); Lac, lactose.

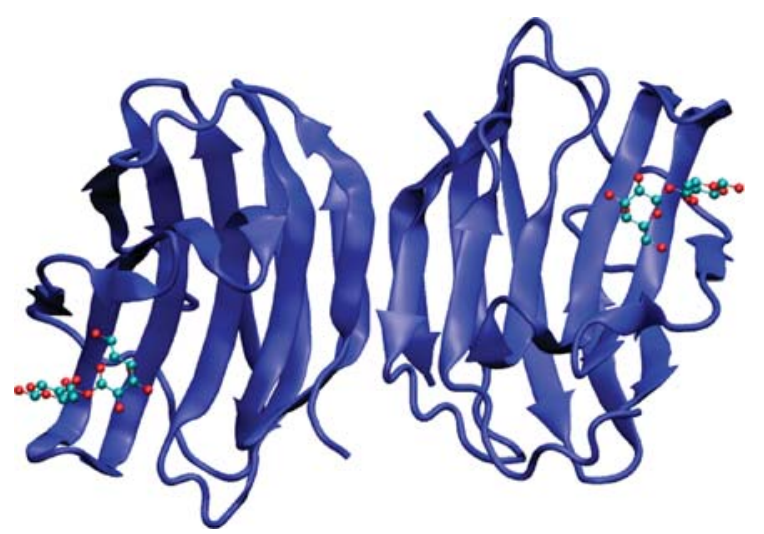

FIGURE 1: Representation of the homodimeric form of human Gal-1 with lactose bound to the carbohydrate recognition domains of each monomer. Protein Data Bank entry 1W6O.

responses (4-7). Through specific interactions with glycoconjugate ligands, Gal-1 has emerged as a powerful regulator of inflammatory responses and tumor progression $(2,5)$. In this context, elucidation of the molecular mechanisms leading to Gal-1-glycan interactions is highly relevant for the design of novel synthetic inhibitors in controlling in vivo activity. Figure 1 shows a ribbon model representation of Gal-1 in its homodimeric form.

The carbohydrate recognition domain (CRD) of Gal-1 consists of a deep channel, an antiparallel $\beta$-sandwich which includes mostly amino acids 44-71. This site is involved in the binding between Gal-1 and a large series of natural in vivo ligands, including glycoproteins with a terminal $\beta$-linked 


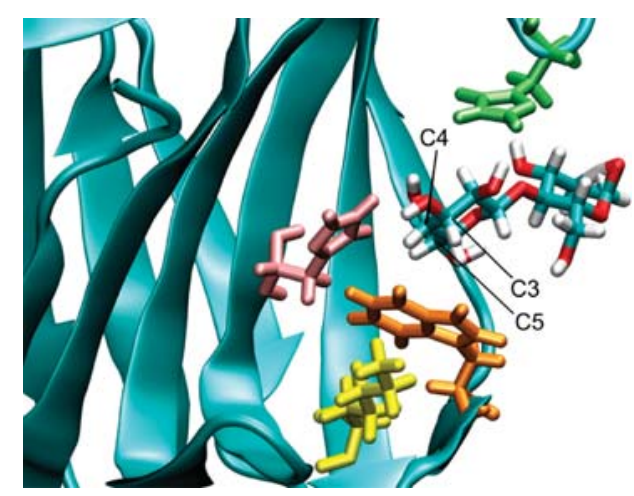

FIGURE 2: Representation of the Gal-1 CRD showing the bound lactose and interacting amino acids: histidine 44 (pink), histidine 52 (green), and tryptophan 68 (orange). Note that one face of the Trp 68 side chain stacks on the sugar ring while the other interacts with Lys 63 (yellow).

galactosyl residue (1), such as laminin, fibronectin, CD45, $\alpha \beta$ integrins, and glycolipids such as GM1 (8-12).

The binding of the galactosyl-terminal residues to the CRD of Gal-1 involves at least two major interactions (13): hydrophilic interactions, via an extensive complementary hydrogen bonding network, and hydrophobic interactions, between sugar rings and aromatic amino acid side chains in the CRD. In particular, Trp68 participates in stacking interactions with $\mathrm{C} 3, \mathrm{C} 4$, and $\mathrm{C} 5$ on the $\beta$-face of the galactose ring, as shown in Figure 2. This fragment appears to be crucial for distinguishing galactose from glucose through its strict preference for the axial $\mathrm{C} 4-\mathrm{OH}$ group, allowing intimate $\mathrm{C}-\mathrm{H}-\pi$-cloud interactions (13).

The binding between lectins and their ligands has been studied by techniques such as isothermal titration microcalorimetry (14), NMR (15), and molecular dynamics simulations (16). Furthermore, galectins and galectinoligosaccharide complexes have been subjects of diverse studies $(17,18)$, some of which examined the molecular basis for ligand recognition (13). The reported crystal structures of Gal-1 in free and ligand-bound states show at least three crucial water molecules participating in a hydrogen bond network in the CRD, two of them being displaced upon ligand binding (19). Recently, by using MD simulations, we identified eight water sites (ws) in the CRD of Gal-1 (20). Water sites were defined as confined space regions close to the protein surface showing a high probability for finding a single water molecule inside them along the simulations. The positions of the ws were defined by the coordinates of the maximum probability point using as a reference surface residues of the protein which are able to interact favorably with the water. Four of the eight ws described in the CRD of Gal-1 were shown to be replaced by the $\mathrm{OH}$ group of the incoming ligand (20).

It is well-known that UV resonance Raman spectroscopy is a powerful tool for monitoring the conformations of proteins $(21-23)$. Excitation at $229 \mathrm{~nm}$ occurs within the electronic transition of the tryptophan aromatic ring. Thus, indole ring vibrations are selectively enhanced and give rise to strong resonance Raman spectra (24). When the indole ring of Trp is exposed to hydrophobic environments, the Trp absorption band red shifts the maximum toward the $229 \mathrm{~nm}$ excitation which increases the enhancement of the Trp Raman bands (22-26). As we will discuss further, the Trp
Raman spectra reveal not only its hydrophobic and hydrophilic environment but also its hydrogen bonding state (27).

In this work, we present the first vibrational study of Gal1, analyzing the UVRR spectra of the Trp68 residue in solvated Gal-1 in the ligand-free and ligand-bound states. We compared the conformations, hydrogen bonding states, and local environment of Trp68 in the presence and absence of the sugar and compared the spectroscopic results to those from MD simulations.

\section{MATERIALS AND METHODS}

Preparation of Recombinant Gal-1. Recombinant human Gal-1 was obtained as previously described (28). Briefly, Escherichia coli BL21(DE3) cells were transformed with a plasmid containing the Lgals1 gene inserted into expression vector $\mathrm{pET}$ (Novagen), and production of recombinant Gal-1 was induced at the $\log$ phase by the addition of $1 \mathrm{mM}$ isopropyl $\beta$-D-thiogalactoside. Cells were separated by centrifugation, washed, and disrupted by sonication. Debris was separated by centrifugation at $10000 \mathrm{~g}$, and soluble fractions were obtained for subsequent purification by affinity chromatography on a lactosyl-Sepharose column (SigmaAldrich). The purification procedure was performed as described previously (29). Recombinant human Gal-1 was stored in a DTT buffer to preserve disulfide protein crosslinks that may affect the activity, as previously described (30).

UV Resonance Raman Experiments at $229 \mathrm{~nm}$. The UV resonance Raman (UVRR) spectrometer has been previously described in detail (31). The $229 \mathrm{~nm}$ excitation was produced by the intracavity frequency doubling of an $\mathrm{Ar}^{+}$laser (Coherent, Innova FReD 300). This UV beam was then focused onto the interior surface of a rotating fused silica NMR tube (Wilmad) filled with $\sim 0.5 \mathrm{~mL}$ of the sample solution. To minimize photodegradation, the laser power was maintained at $100 \mu \mathrm{W}$. The solution was stirred extensively with a $0.5 \mathrm{~mm}$ long magnetic stirring bar. Additionally, a Teflon wire with a flat tip was placed inside the tube just above the area of incident light to induce turbulence. The lack of degradation of the sample under these conditions was ensured within the first $10 \mathrm{~min}$ of data collection. No differences were observed in the intensity of the data collected in the 10th minute with respect to those obtained in the 1 st minute. A $180^{\circ}$ backscattering geometry was used for the Raman measurements.

In all experiments, we used $1 \mathrm{mg} / \mathrm{mL}$ Gal- 1 in a phosphatebuffered saline solution, which yields a Trp concentration of $70 \mu \mathrm{M}$. Samples of the Gal-1-Lac complex were prepared by adding lactose to a final concentration of $30 \mathrm{mM}$. As the value of the Gal-1 affinity constant reported for lactose $\left(K_{\mathrm{b}}\right)$ at room temperature is $3.25 \times 10^{3} \mathrm{M}^{-1}(19)$, the lactose concentration used ensures that more than $97 \%$ of the protein is bound. The Raman spectra of Gal-1 and the Gal-1-Lac complex in the presence of $0.2 \mathrm{M} \mathrm{NaClO}_{4}$, used as an internal standard, were also recorded.

Molecular Dynamics Simulations. Gal-1 coordinates were retrieved from the Protein Data Bank, entry $1 \mathrm{~W} 6 \mathrm{~N}$ for the unbound protein (X-ray, $1.65 \AA$ resolution) and entry $1 \mathrm{~W} 6 \mathrm{O}$ for the Gal-1-Lac complex. The C2S mutant (wild-type cysteine in position 2 replaced with a serine) was used, due to the availability of crystallographic data for the mutant 


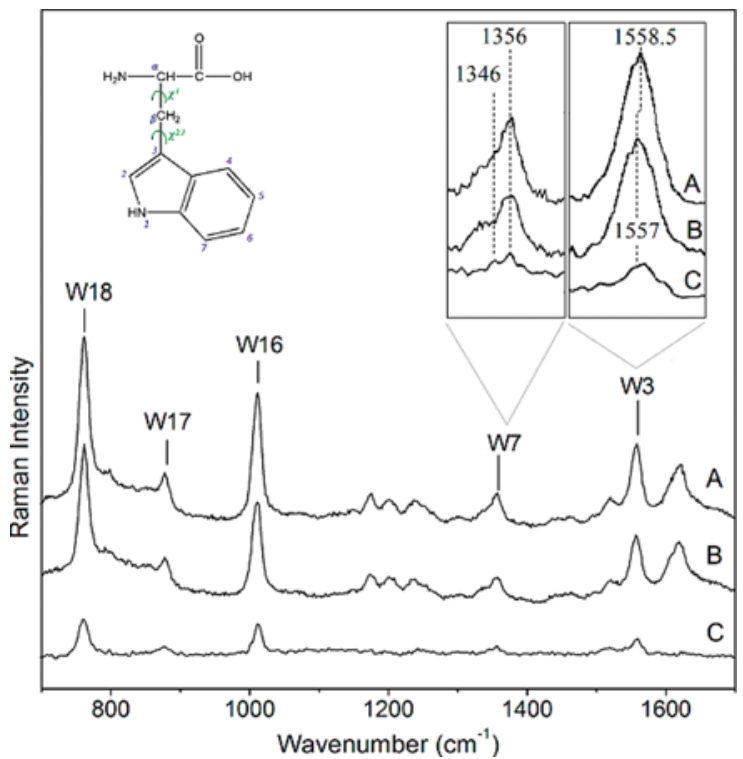

FIGURE 3: Resonance Raman spectra at $229 \mathrm{~nm}$ of (A) the Gal1-Lac complex and (B) Gal-1. The intensities were normalized with respect to the Tyr Y8a band. (C) Difference spectrum obtained by subtracting spectrum $B$ from spectrum A. Note that the difference spectrum shows only Trp signals. In the insets, the W3 band shift can be better visualized, and it is clearly shown that the W7 $I_{1356} /$ $I_{1346}$ ratio decreases from spectrum A to B (Gal-1 bound to unbound state, respectively).

protein and the mutant bound to different ligands $(13,20)$. Since the $\mathrm{C} 2 \mathrm{~S}$ mutation is far from the CRD, we assume that it will not affect binding.

In both cases, all crystallographic water molecules were deleted, and a single subunit was then solvated with threesite point charge-modeled (TIP3P) water molecules in an octahedral box. The MD simulations were performed using the AMBER 8 (32) package of programs, with the PARM99 set of parameters (33) and the GLYCAM-04 parameters for carbohydrates (34).

The equilibration protocol consisted of performing 500cycle runs of minimization to remove initial unfavorable contacts, followed by 100 ps simulations, during which systems were slowly heated from $0 \mathrm{~K}$ to the desired temperature of $300 \mathrm{~K}$. The pressure was then equilibrated at $1 \mathrm{~atm}$ over $200 \mathrm{ps}$.

All simulations used the periodic boundary condition approximation and Ewald summation method, with an $8 \AA$ cutoff. The SHAKE algorithm was applied to all hydrogencontaining bonds. The MD simulations were performed at $300 \mathrm{~K}$, using a time step of $2 \mathrm{fs}$. All atoms in the simulated systems were allowed motional freedom. Snapshots of the coordinates were saved every $2 \mathrm{ps}$, over a total $20 \mathrm{~ns}$ of trajectory. The resulting 10000 instantaneous configurations saved on disk for each system were then analyzed.

In addition, a $5 \mathrm{~ns}$ simulation was performed for an isolated and solvated Trp amino acid. The MD simulation was performed using the same computational setup described above.

\section{RESULTS AND DISCUSSION}

Excitation of Trp at $229 \mathrm{~nm}$ Changes upon Binding. Figure 3 shows the $229 \mathrm{~nm}$ excited resonance Raman spectra of Gal-1 and the Gal-1-Lac complex and the difference

\begin{tabular}{lcrr}
\hline Table 1: $\operatorname{Trp} \mathrm{C} 2=\mathrm{C} 3-\mathrm{C} \beta-\mathrm{C} \alpha \chi^{2,1}$ Dihedral Angles (degrees) \\
\hline & crystal $^{a}$ & $\mathrm{MD}^{b}$ & Raman $^{c}$ \\
\hline$\chi^{2,1}(\mathrm{Gal}-1)$ & 120.3 & 96.8 & 114.4 \\
$\chi^{2,1}(\mathrm{Gal}-1-\mathrm{Lac})$ & 119.3 & 101.2 & $\sim 120.0$ \\
$\Delta \chi^{2,1}$ & -1.0 & 4.4 & $\sim 5.6$ \\
\hline
\end{tabular}

${ }^{a}$ Extracted from the crystal structures (19). ${ }^{b}$ Average value over the snapshots from the MD simulations of each system. ${ }^{c}$ Evaluated according to ref 36 .

spectrum. The dominant bands in the spectra at 762,877 , 1011, 1356, 1557, and $1622 \mathrm{~cm}^{-1}$ derive from Trp and Tyr residues of Gal-1 which are assigned to Trp W18, W17, $\mathrm{W} 16$, W7, and W3 vibrations and a Tyr Y8a vibration, respectively. Since lactose is not resonance-enhanced for 229 $\mathrm{nm}$ excitation, it does not make any contribution to the Raman spectra.

Binding of lactose results in an intensity increase of the Trp bands, which is clearly evident in the difference spectrum (Figure 3C). No Tyr band features appear in the difference spectrum, as expected since Tyr residues in Gal-1 are not localized in the binding site and are not directly involved in binding.

The intensity increase of the Trp bands of the Gal-1-Lac complex supports the idea that the incoming ligand excludes water from the indole ring surroundings, giving rise to a more hydrophobic environment around the Trp.

In separate experiments, we evaluated the usage of a salt as an internal standard to quantitatively measure Raman intensities. While many salts were not considered due to interactions altering the protein function (19), the commonly used (35) sodium perchlorate was then evaluated. We measured the UVRR spectra of Gal-1 and the Gal-1-Lac complex in the presence and absence of perchlorate and found that perchlorate affects the binding of lactose to Gal1 , since a much smaller Trp band intensity increase occurs in the presence of perchlorate. We presume that perchlorate oxidizes the thiol groups of cysteines, causing a decreased affinity for glycan ligands, as reported previously (19).

Tryptophan Side Chain Orientation. The $1557 \mathrm{~cm}^{-1}$ Raman peak is derived from the Trp W3 indole in-plane ring vibration, which has a large component of $\mathrm{C} 2=\mathrm{C} 3$ stretching. The frequency of $\mathrm{W} 3$ depends on the torsion angle $\left(\chi^{2,1}\right)$ involving the $\mathrm{C} 2=\mathrm{C} 3-\mathrm{C} \beta-\mathrm{C} \alpha$ linkage. Takeuchi found a quantitative relationship between $\chi^{2,1}$ and the W3 frequency, and from this relationship, we can estimate a $\chi^{2,1}$ angle for non-lactose-bound Trp 68 in Gal-1 of $114.4^{\circ}$, which is in agreement with the value obtained from the crystal structure $\left(120.3^{\circ}\right)$. Once lactose binds, the W3 frequency upshifts $\sim 1.5$ $\mathrm{cm}^{-1}$, resulting in an angle of $120^{\circ}$, which is close to the upper limit of the W3 frequency $-\chi^{2,1}$ relationship (36). Table 1 lists the $\chi^{2,1}$ torsion angles for Gal-1 and the Gal-1-Lac complex obtained from the Raman data, the crystal structure, and the MD simulations.

MD simulations predict a lower $\chi^{2,1}$ angle of $\sim 100^{\circ}$ and a small increase of $4.4^{\circ}$ for this angle in the Gal-1-Lac complex. The Raman, crystal, and MD results all indicate a lack of significant conformational changes around the $\mathrm{C} 3-\mathrm{C} \beta$ bond for Gal-1 upon lactose binding.

Nitrogen Hydrogen Bonding State. The Trp W17 vibration involves a benzene $v_{12}$-like vibration from the phenyl ring which couples to $\mathrm{N}-\mathrm{H}$ bond motion (27). The W17 frequency is sensitive to the hydrogen bonding state of the 


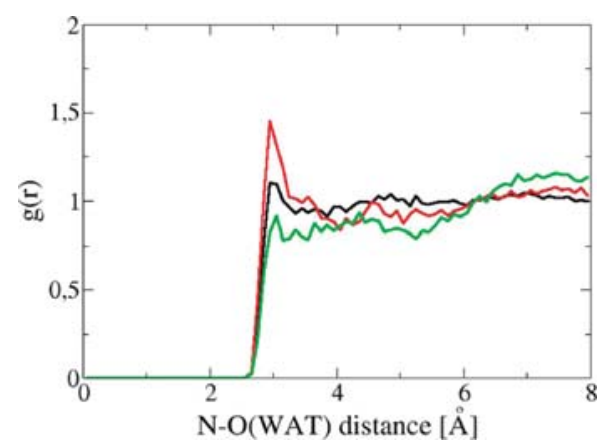

FIGURE 4: Water radial distribution function for the nitrogen of the side chain of Trp68 of Gal-1 (black), Trp68 of the Gal-1-Lac complex (red), and the isolated Trp in water (green), obtained from MD simulations.

indole $\mathrm{NH}$ group (37). In the absence of $\mathrm{NH}$ group hydrogen bonding, the $\mathrm{W} 17$ band appears at $883 \mathrm{~cm}^{-1}$, while strong hydrogen bonding downshifts the band to $871 \mathrm{~cm}^{-1}$. In the spectrum of Gal-1, this vibrational mode is observed in the middle of the frequency range $\left(877 \mathrm{~cm}^{-1}\right)$, indicating that the indole $\mathrm{NH}$ group is moderately hydrogen bonded to a proton acceptor (36). This is consistent with both the MD simulation results which indicate the presence of a ws interacting with the Trp $\mathrm{NH}$ group in the solvated protein (20) and the crystal structure of Gal-1 where the indole nitrogen appears oriented toward the protein surface and is close to a water molecule ( $\mathrm{N}-\mathrm{O}$ distance of $3.72 \AA$ ) (19). No W17 band frequency change is observed upon lactose binding, suggesting that the lactose does not directly interact with the indole $\mathrm{NH}$ group. The moderate strength of the $\mathrm{NH}$ hydrogen bond in the lactose-bound state is consistent with the presence of surface water molecules; in the crystal structure of the complex, two surface water molecules are observed in the vicinity of Trp68 $(\mathrm{N}-\mathrm{O}$ distances of 2.87 and $2.99 \AA$ ) (19).

Figure 4 shows the radial distribution function, $g(r)$, for water molecules around the indole nitrogen of the Trp in the three situations: as an isolated amino acid in water, as the Trp68 residue in Gal-1, and in the Gal-1-Lac complex. While the nitrogen of the isolated Trp does not exhibit a structured solvation shell, Trp68 of both Gal-1 and the Gal1 -Lac complex shows a peak in $g(r)$ at $2.9 \AA$, corresponding to water molecules involved in hydrogen bonds to the nitrogen. The higher probability of finding localized water molecules around the indole nitrogen in the Gal-1-Lac complex observed in MD simulations is in agreement with the presence of water molecules close to that nitrogen observed in the crystallographic structure.

Hydration Environment. The W7 band provides further insight into the dependence of Trp hydration upon lactose binding. The W7 band of Trp usually splits into a doublet at $\sim 1360$ and $1340 \mathrm{~cm}^{-1}$ due to Fermi resonance between a fundamental band of an in-plane vibration and one or more combination out-of-plane vibrations. The intensity ratio of the doublet changes with the environmental hydrophobicity around the indole ring: the stronger the hydrophobicity, the higher the $I_{1360} / I_{1340}$ intensity ratio $(37,38)$. The frequencies of the out-of-plane vibrations are affected by hydrophobic interactions of the indole ring, which alters the doublet relative intensity ratio. In the UVRR spectra of Gal-1 and the Gal-1-Lac complex, the main component of the W7 doublet is observed at $1356 \mathrm{~cm}^{-1}$ with a broad shoulder

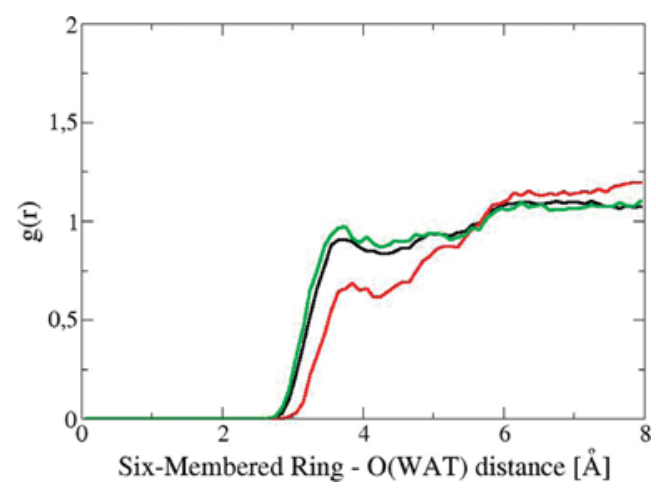

FIGURE 5: Water radial distribution function for the six-membered ring of the side chain of Trp68 of Gal-1 (black), Trp68 of the Gal1-Lac complex (red), and the isolated Trp in water (green), obtained from MD simulations.
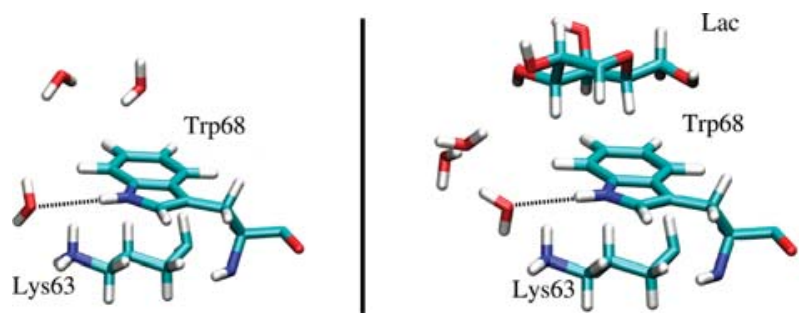

FIGURE 6: Instantaneous configurations showing interactions between Trp68 of Gal-1 and Lys63 and (a) water molecules in the unbound state (left) and (b) the galactosyl moiety of lactose bound to the CRD (right).

indicating the second component. In the difference spectrum, both Fermi resonance components are clearly observed at 1356 and $1346 \mathrm{~cm}^{-1}$. The change to a more hydrophobic environment for the Trp in the binding site as a consequence of lactose addition is supported well by the extra intensity of the $1356 \mathrm{~cm}^{-1}$ W7 Fermi doublet component over the $1346 \mathrm{~cm}^{-1}$ component.

The spectral evidence observed for the hydration environment of the Trp68 may also be explained in terms of the water radial distribution functions for the side chain of the residue, shown in Figure 5. The $g(r)$ was computed for water molecules around the center of mass determined by the heavy atoms of the six-membered ring.

The calculated $g(r)$ for the side chain of Trp68 in unbound protein is similar to that for the Trp in a water solution, while they show a significant difference with the $g(r)$ calculated for Trp68 in the Gal-1-Lac complex. It is evident from the functions that the six-membered ring undergoes a noticeable change in its hydration environment, decreasing its level of solvent exposure when the lactose accesses the CRD. Figure 6 shows that the Trp68 side chain in Gal-1 is surrounded by lysine 63 with a significant solvent accessibility on one of its faces, while in the Gal-1-Lac complex, this residue is involved in the stacking interaction with the galactosyl terminus of the ligand.

\section{CONCLUSIONS}

Our results indicate that the addition of lactose to Gal-1 does not significantly affect the Trp residue conformation in the binding site since no significant frequency shifts occur for conformation marker W3 and hydrogen bonding marker W17 bands. These results are consistent with MD modeling studies. However, as confirmed by the MD simulations, 
lactose binding results in a more hydrophobic environment of the Trp residue. Subtle solvent reorganization was evaluated by the proposed methodologies. In this regard, both Raman signal intensities and water radial distribution functions confirm that the presence of ligand causes displacement of water molecules in the surroundings of the Trp side chain, while no substantial change was observed in the hydrogen bond network of the nitrogen of the indolyl group upon binding.

\section{ACKNOWLEDGMENT}

S.D.L. thanks Zeeshan Ahmed, Kan Xiong, Diego Croci, and Germán Bianco for substantial help with experiments and Marcelo A. Martí and Darío A. Estrin for important discussions and computational facilities.

\section{REFERENCES}

1. Cooper, D. N. W., and Barondes, S. H. (1999) God must love galectins; he made so many of them. Glycobiology 9, 979-984.

2. Cho, M., and Cummings, R. D. (1995) Galectin-1, a $\beta$-galactosidebinding lectin in Chinese hamster ovary cells. I. Physical and chemical characterization. J. Biol. Chem. 270, 5198-5206.

3. Toscano, M. A., Ilarregui, J. M., Bianco, G. A., Campagna, L., Croci, D. O., Salatino, M., and Rabinovich, G. A. (2007) Dissecting the pathophysiologic role of endogenous lectins: Glycan-binding proteins with cytokine-like activity? Cytokine Growth Factor Rev. 18, 57-71.

4. Toscano, M., Bianco, G. A., Ilarregui, J. M., Croci, D. O., Correale, J., Hernandez, J. D., Zwirner, N. W., Poirier, F., Riley, E. M., Baum, L. G., and Rabinovich, G. A. (2007) Differential glycosylation of TH1, TH2 and TH-17 effector cells selectively regulates susceptibility to cell death. Nat. Immunol. 8, 825-834.

5. Rabinovich, G. A., Toscano, M., Jackson, D. A., and Vasta, G. (2007) Functions of cell surface galectin-glycoprotein lattices. Curr Opin. Struct. Biol. 17, 513-520.

6. Rabinovich, G. A. (2005) Galectin-1 as a potential cancer target. Br. J. Cancer 92, 1188-1192.

7. Rabinovich, G. A., Liu, F. T., Hirashima, M., and Anderson, A. (2007) An emerging role for lectins in tuning the immune response: Lessons from experimental models of inflammatory disease, autoimmunity and cancer. Scand. J. Immunol. 66, 143-158.

8. Elola, M. T., Wolfenstein-Todel, Ç., Troncoso, M. F., Vasta, G. R., and Rabinovich, G. A. (2007) Galectins: Matricellular glycanbinding proteins linking cell adhesion, migration and survival. Cell. Mol. Life Sci. 64, 1679-1700.

9. Andre, S., Kojima, S., Yamazaki, N., Fink, C., Kaltner, H., Kayser, K., and Gabius, H. J. (1999) Galectins-1 and -3 and their ligands in tumor biology. Non-uniform properties in cell-surface presentation and modulation of adhesion to matrix glycoproteins for various tumor cell lines, in biodistribution of free and liposome-bound galectins and in their expression by breast and colorectal carcinomas with/without metastatic propensity. J. Cancer Res. Clin. Oncol. $125,461-474$

10. Walzel, H., Schulz, U., Neels, P., and Brock, J. (1999) Galectin1, a natural ligand for the receptor-type protein tyrosine phosphatase CD45. Immunol. Lett. 67, 193-202.

11. Gu, M., Wang, W., Song, W. K., Cooper, D. N., and Kaufman, S. J. (1994) Selective modulation of the interaction of $\alpha 7 \beta 1$ integrin with fibronectin and laminin by L-14 lectin during skeletal muscle differentiation. J. Cell Sci. 107, 175-181.

12. Kopitz, J., von Reitzenstein, C., Burchert, M., Cantz, M., and Gabius, H. J. (1998) Galectin-1 is a major receptor for ganglioside GM1, a product of the growth-controlling activity of a cell surface ganglioside sialidase, on human neuroblastoma cells in culture. J. Biol. Chem. 273, 11205-11211.

13. Ford, M. G., Weimar, T., Kölhi, T., and Woods, R. J. (2003) Molecular Dynamics Simulations of Galectin-1-oligosaccharides Complexes Reveal the Molecular Basis of Ligand Diversity. Proteins: Struct., Funct., Genet. 53, 229-240.

14. Ahmad, N., Gabius, H., Sabesan, S., Oscarson, S., and Brewer, C. F. (2004) Thermodynamic binding studies of bivalent oligosaccharides to galectin-1, galectin-3, and the carbohydrate recognition domain of galectin-3. Glycobiology 14, 817-825.
15. Asensio, J. L., Siebert, H.-C., von der Lieth, C.-W., Laynez, J., Bruix, M., Soedjanaamadja, U. M., Beintema, J. J., Cañada, F. J., Gabius, H., and Jiménez-Barbero, J. (2000) NMR Investigations of Protein-Carbohydrate Interactions: Studies on the Relevance of Trp/Tyr Variations in Lectin Binding Sites as Deduced from Titration Microcalorimetry and NMR Studies on Hevein Domains. Determination of the NMR Structure of the Complex Between Pseudohevein and $\mathrm{N}, \mathrm{N}^{\prime}, \mathrm{N}^{\prime}$-Triacetylchitotriose. Proteins: Struct., Funct., Genet. 40, 218-236.

16. Clarke, C., Woods, R. J., Gluska, J., Cooper, A., Nutley, M. A., and Boons, G. (2001) Involvement of Water in CarbohydrateProtein Binding. J. Am. Chem. Soc. 123, 12238-12247.

17. Sörme, P., Arnoux, P., Kahl-Knutsson, B., Leffler, H., Rini, J. M., and Nilsson, U. J. (2005) Structural and Thermodynamic Studies on Cation- $\pi$ interactions in Lectin-Ligand Complexes: HighAffinity Galectin-3 Inhibitors through Fine-Tuning of an ArginineArene Interaction. J. Am. Chem. Soc. 127, 1737-1743.

18. Rabinovich, G. A., Cumashi, A., Bianco, G. A., Ciavardelli, D., Iurisci, I., D’Egidio, M., Piccolo, E., Tinari, N., Nifantiev, N., and Iacobelli, S. (2005) Synthetic lactulose amines: Novel class of anticancer agents that induce tumor-cell apoptosis and inhibit galectin-mediated homotypic cell aggregation and endothelial cell morphogenesis. Glycobiology 16, 210-220.

19. Lopez-Lucendo, M. F., Solis, D., Andre, S., Hirabayashi, J., Kasai, K., Kaltner, H., Gabius, H. J., and Romero, A. (2004) Growthregulatory human galectin-1: Crystallographic characterisation of the structural changes induced by single-site mutations and their impact on the thermodynamics of ligand binding. J. Mol. Biol. 343, 957-970.

20. Di Lella, S., Marti, M. A., Alvarez, R. M. S., Estrin, D. A., and Díaz Ricci, J. C. (2007) Characterization of the Carbohydrate Recognition Domain of Galectin-1 in Terms of Solvent Occupancy. J. Phys. Chem. B 111, 7360-7366.

21. Asher, S. (1993) UV Resonance Raman spectroscopy for analytical, physical, and biophysical chemistry. Anal. Chem. 65, 201-210.

22. Chi, Z., and Asher, S. (1998) UV resonance Raman determination of protein acid denaturation: Selective unfolding of helical segments of horse myoglobin. Biochemistry 37, 2865-2872.

23. Ahmed, Z., Beta, I. A., Mikhonin, A. V., and Asher, S. (2005) UV-Resonance Raman Thermal Unfolding Study of Trp-Cage Shows That It Is Not a Simple Two-State Miniprotein. J. Am. Chem. Soc. 127, 10943-10950.

24. Chi, Z., and Asher, S. (1998) UV Raman Determination of the Environment and Solvent Exposure of Tyr and Trp Residues. $J$. Phys. Chem. B 102, 9595-9602.

25. Kamlet, M. J., Abbound, J.-L. M., and Taft, R. W. (1981) An examination of linear solvation energy relationships. Prog. Phys. Org. Chem. 13, 485-630.

26. Efremov, R. G., Feofanov, A. V., and Nabiev, I. R. (1992) Effect of hydrophobic environment on the resonance Raman spectra of tryptophan residues in proteins. J. Raman Spectrosc. 23, 69-73.

27. Takeuchi, H., and Harada, I. (1986) Normal Coordinate Analysis of the Indole Ring. Spectrochim. Acta 42, 1067-1078.

28. Hirabayashi, J., Hashidate, T., Arata, Y., Nishi, N., Nakamura, T., Hirashima, M., Urashima, T., Oka, T., Futai, M., and Muller, W. E. (2002) Oligosaccharide specificity of galectins: A search by frontal affinity chromatography. Biochim. Biophys. Acta 1572, 232-254.

29. Barrionuevo, P., Beigier-Bompadre, M., Ilarregui, J. M., Toscano, M., Bianco, G. A., Isturiz, M. A., and Rabinovich, G. A. (2007) A novel function for galectin-1 a the crossroad of innate and a adaptive immunity: Galectin-1 regulates monocyte/macrophage physiology through a nonapoptotic ERK-dependent pathway. J. Immunol. 178, 436-445.

30. Pace, K. E., Hahn, H. P., and Baum, L. G. (2003) Preparation of recombinant human galectin-1 and use in $\mathrm{T}$ cell death assays. Methods Enzymol. 363, 499-518.

31. Asher, S. A., Bormett, R. W., Chen, X. G., Lemmon, D. H., Cho, N., Peterson, P., Arrigoni, M., Spinelli, L., and Cannon, J. (1993) UV Resonance Raman Spectroscopy Using a New CW Laser Source: Convinience and Experimental Simplicity. Appl. Spectrosc. 47, 628-633.

32. Case, D. A., Darden, T. A., Cheatman, T. E., III, Simmerling, C. L., Wang, J., Duke, R. E., Luo, R., Merz, D. M., Wang, B., Pearlman, D. A., Crowley, M., Brozell, S., Tsui, V., Gohlke, H., Mongan, J., Hornak, V., Cui, G., Beroza, P., Schafmeister, C., Caldwell, J. W., Ross, W. S., and Kollman, P. A. (2004) AMBER 8, University of California, San Francisco.

33. Cheatham, T. E., Cieplak, P., and Kollman, P. A. (1999) J. Biomol. Struct. Dyn. 16, 845-862. 
34. Case, D. A., Cheatham, T. E., Darden, T. A., Gohlke, H., Luo, R., Merz, K. M. J., Onufriev, A., Simmerling, C., Wang, B., and Woods, R. J. (2005) The Amber biomolecular simulation programs. J. Comput. Chem. 26, 1668-1688.

35. Dudik, J. M., Johnson, C. R., and Asher, S. A. (1985) UV Resonance Raman studies of acetone, acetamide, and $\mathrm{N}$-methylacetamide: Models for the peptide bond. J. Phys. Chem. 89, 3805-3814.

36. Takeuchi, H. (2003) Raman Structural Markers of Tryptophan and Histidine Side Chains in Proteins. Biopolymers 72, 305-317.
37. Miura, T., Takeuchi, H., and Harada, I. (1988) Characterization of individual tryptophan side chains in proteins using Raman spectroscopy and hydrogen-deuterium exchange kinetics. Biochemistry 27, 88-94.

38. Harada, I., Miura, T., and Takeuchi, H. (1986) Origin of the doublet at 1360 and $1340 \mathrm{~cm}^{-1}$ in the Raman spectra of tryptophan and related compounds. Spectrochim. Acta 42, 307-312.

BI801855G 